\title{
Magnetic Forces Investigation of Bulk HTS over Permanent Magnetic Guideway under Different Lateral Offset with 3D-Model Numerical Method
}

\author{
Yiyun Lu and Qiaohong Dang \\ Luoyang Institute of Science and Technology, Henan, Luoyang 471023, China \\ Correspondence should be addressed to Yiyun Lu, luyiyun6666@vip.sohu.com
}

Received 4 July 2012; Accepted 27 September 2012

Academic Editor: Mark Blamire

Copyright ( $) 2012$ Y. Lu and Q. Dang. This is an open access article distributed under the Creative Commons Attribution License, which permits unrestricted use, distribution, and reproduction in any medium, provided the original work is properly cited.

\begin{abstract}
Magnetic forces of a cylinder shape bulk high-temperature superconductor (HTS) over a permanent magnet guideway (PMG) are studied mathematically. One cylindrical bulk HTS with a diameter of $30 \mathrm{~mm}$ and $15 \mathrm{~mm}$ in height is used. Two types of PMG are employed for external magnetic fields consideration. The relationship of magnetic forces of bulk HTS under different lateral offsets over PMG is studied with 3D-model finite element method (FEM). The calculation results show that the maximum magnetic levitation force of bulk HTS over PMG is tightly related to the applied magnetic field distribution. For the symmetrical PMG, the maximum magnetic levitation force decreases linearly with the increase of lateral offset of the bulk sample. For the Halbach PMG, when lateral offset changes from $0 \mathrm{~mm}$ to $25 \mathrm{~mm}$, the maximum magnetic levitation force increases with the increase of lateral offset of the bulk HTS. When the lateral offset exceeds the center of the Halbach by $25 \mathrm{~mm}$, the maximum levitation force decreases rapidly with the increase of the lateral offset of the bulk sample.
\end{abstract}

\section{Introduction}

Since the discovery of bulk high temperature superconductor (HTS) which can stably levitate above permanent magnet (PM), the magnetic levitation transportation system has attracted many researchers focus on its potential application $[1,2]$. Besides the first man loading HTS Maglev test vehicle [1], several high temperature superconducting Maglev test vehicle had been successfully developed [3-6]. In the last two decades, researchers have done many experiments to try to understand the magnetic levitation system composed of bulk HTS and PM or PMs. Deng experimentally compared the cost performance of YBCO bulks over two types of permanent magnet guideway (PMG) by magnetic levitation and guidance forces [7]. Zhang experimentally researched the magnetic levitation force relaxation characteristics of bulk HTS over PM under different temperatures [8]. He made several destructive experiments for the study of levitation force characteristics of YBCO bulk with a linear notch over one cylindrical PM $[9,10]$. Liu has researched the magnetic levitation force decay characteristics of YBCO bulk over PMG while the bulk sample is applied with external AC magnetic field [11-13]. Lu experimentally measures magnetic force stiffness of YBCO bulk arrays over two types of PMG [14].

During the bulk HTS Maglev system optimization design, many researchers focus on the peak value of magnetic levitation and guide forces of bulk HTS. The maximum magnetic force is tightly related with bulk HTS critical current density, flux pinning ability, and the peak value of applied magnetic fields. Many practice prototype Maglev test vehicles involve bulk HTS arrays and PMG. How to arrange the bulk HTS arrays above the PMG and the magnetic field distribution induced by the PMG also needs to be investigated. For cylindrical bulk HTS arrays, two main arrangements are often used, as Figures 1(a) and 1(b) show. The PMG also needs to be carefully considered for optimal design. Two main factors are involved during the design progress. One is the peak value of magnetic fields generated by the PMG, the other is the distribution of the magnetic 


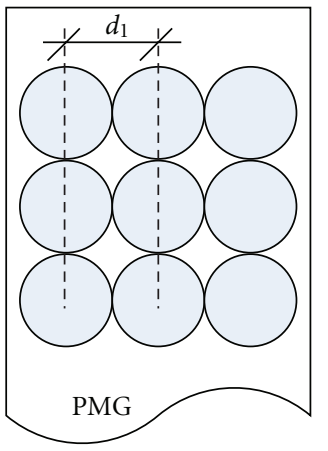

(a)

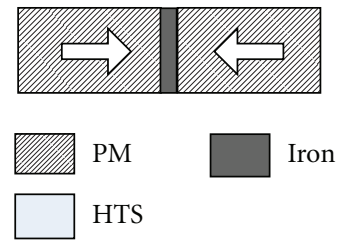

(c)

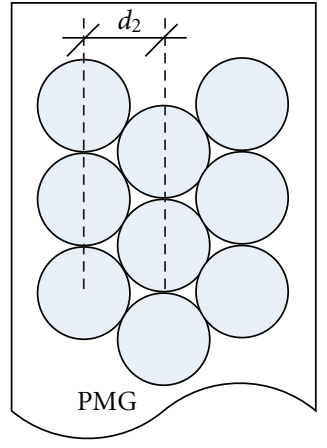

(b)

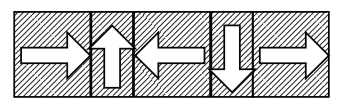

(d)
FIgure 1: The two cylindrical bulk HTS arrays above PMG and the schematic diagram of two types PMG (a) bulk HTS side-by-side array (b) bulk HTS stagger array (c) symmetrical PMG (d) Halbach PMG.

fields. Usually the two factors are difficult to weigh. Figures 1 (c) and 1(d) show the construction diagram of two types PMG.

In the paper, the influence of magnetic field distribution of PMG on the magnetic levitation force of bulk HTS is investigated. The investigation is carried out by the method that magnetic levitation forces of bulk HTS with different lateral offsets over PMG are simulated with 3Dmodel numerical method. The lateral offset of the bulk HTS over PMG increases from $0 \mathrm{~mm}$ to $30 \mathrm{~mm}$ with the step size equal to $5 \mathrm{~mm}$. The proposed 3D-model method uses two virtual bulk samples for mathematical descriptions of high- $T_{c}$ superconducting electrical field and current density nonlinear problem [15]. The simulation results show that the maximum value of magnetic levitation forces of different lateral offsets of bulk HTS over PMG is tightly related to the applied magnetic field distribution. For the symmetrical PMG, the maximum value of magnetic levitation forces decreases linearly with the lateral offset increasing of the bulk HTS. For the Halbach PMG, when the lateral offset changes from $0 \mathrm{~mm}$ to $25 \mathrm{~mm}$, the maximum value of magnetic levitation forces of the bulk HTS increases with the lateral offset increasing. When the lateral offset exceeds the center of the Halbach by $25 \mathrm{~mm}$, the maximum value decreases rapidly with the increase of the lateral offset of the bulk sample.

\section{Basic Equations for 3D-Model}

For the proposed numerical method introduced in this section, one cylindrical shape bulk HTS levitated above PMG is considered, as Figure 2 shows. The calculation regions

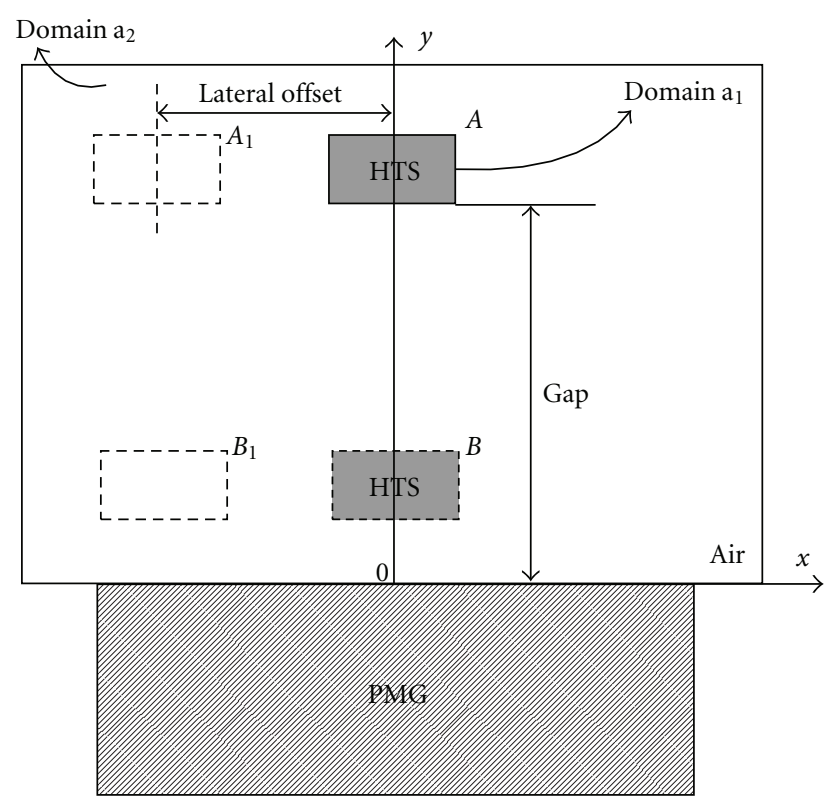

FIGURE 2: The cross section of bulk HTS-PMG levitation 3D-model.

include domain $\mathrm{a}_{1}$ and domain $\mathrm{a}_{2}$. The two calculation regions are satisfied for Maxwell-Ampere's law and Faraday's law:

$$
\begin{gathered}
\nabla \times \mathbf{H}=\mathbf{J} \\
\nabla \times \mathbf{E}=-\frac{\partial \mathbf{B}}{\partial t} .
\end{gathered}
$$

For the quasi-approximation problem, we assume no displacement currents are considered. Here $\mathbf{E}$ and $\mathbf{H}$ are the electrical field and magnetic field of the calculation regions, respectively.

The $\mathbf{B}-\mathbf{H}$ constitutive law:

$$
\mathbf{B}=\mu \mathbf{H} .
$$

In order to simplify the equation derivation, a virtual Ohm's law is considered for all the calculation regions:

$$
\mathbf{E}=\frac{1}{\sigma} \mathbf{J} .
$$

For the calculation region of domain $a_{1}$ which corresponds to bulk HTS, E-J power law is used to describe the EJ constructive relation of high- $T_{c}$ superconducting materials as follows:

$$
\mathbf{E}=E_{c 0}\left(\frac{|\mathbf{J}|}{J_{c}}\right)^{n} \frac{\mathbf{J}}{|\mathbf{J}|} .
$$

From (5) we can see that vector $\mathbf{E}$ and $\mathbf{J}$ have the same direction. Here $E_{c 0}$ is material and temperature dependent constant and $J_{c}$ is the critical current density of the bulk HTS.

The virtual conductivity of domain $\mathrm{a}_{1}$ can be derived by (4) and (5) as follows:

$$
\frac{1}{\sigma_{s c}}=\frac{|\mathbf{E}|}{|\mathbf{J}|}=\frac{E_{c 0}}{J_{c}}\left(\frac{|\mathbf{J}|}{J_{c}}\right)^{n-1} .
$$


Critical current density in the bulk YBCO interior along a-b plane is about two times than that along $c$-axis. Thus for high- $T_{c}$ superconducting nonlinear materials problem, the critical current density $J_{c}$ alongside $c$-axis is considered one third of the critical current density flowing alongside $a-b$ plane of bulk HTS. We assume that the calculation region of domain-a $a_{1}$ (see Figure 2 ) is mathematically composed by two parts: one is a homogeneous bulk HTS and the other only $c$ axis oriental conductivity which is considered not equal to zero. Based on the assumption, consider (3) and (4) we can get the equation:

$$
\frac{1}{\sigma_{s c}(\mathbf{J})} \nabla \times\left\{\left[\begin{array}{c}
J_{x} \\
J_{y} \\
J_{z}
\end{array}\right]+\left[\begin{array}{c}
0 \\
2 J_{y} \\
0
\end{array}\right]\right\}=-\mu \frac{\partial \mathbf{H}}{\partial t} .
$$

For all the calculation regions of Figure 2, substitute (1) into (7) and consider the component $J_{y}$ of current density $\mathbf{J}$ can be expressed as $J_{y}=\partial H_{x} / \partial z-\partial H_{z} / \partial x$, the governing equation can be successfully derived from

$$
\mu \frac{\partial}{\partial t} \mathbf{H}+\nabla \times \frac{1}{\sigma} \nabla \times \mathbf{H}+\frac{\lambda}{\sigma} \nabla \times \mathbf{Q}=0 .
$$

Here $\sigma=\sigma_{\text {air }}, \lambda=0$ for domain $\mathrm{a}_{2}$ and $\sigma=\sigma_{\text {sc }}(\mathbf{J}), \lambda=1$ for domain $a_{1}$ (see Figure 2) and define vector $\mathbf{Q}$ as $\mathbf{Q}=$

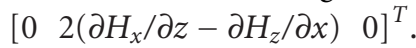

Formulation (8) is a time-varying electromagnetic partial differential equation derived from Faraday's law, Ampere's law, and E-J power law.

The outer boundary of domain $\mathrm{a}_{2}$ is a dynamic boundary. Here we use a time dependence function to describe

$$
\mathbf{H}_{\mathrm{a}_{2}}(r, t)=f_{\mathrm{a}_{2}}(r, t) .
$$

The detailed numerical method and implementation procedure here are the same as those in our previous research (please see [15]).

After the magnetic fields and current density distribution are resolved, the magnetic forces acting on the bulk HTS can be obtained by

$$
\mathbf{F}_{\mathrm{mag}}=\int_{V} \mathbf{J} \times \mathbf{B}_{\mathrm{ex}} d v
$$

Here $V$ represents the volume of the bulk HTS, $\mathbf{J}$ is the induced current density, and $\mathbf{B}_{\mathrm{ex}}$ is the applied external magnetic field.

\section{Levitation Force and Guidance Force Simulation of Bulk HTS over PMG}

The prototype magnetic levitation system is simulated with the proposed methods. As Figure 2 shows, the simple levitation system composes of a cylindrical bulk HTS and a PMG. The mini gap from position B to the top surface of the PMG is $3 \mathrm{~mm}$. The cylindrical bulk HTS is $30 \mathrm{~mm}$ in diameter and $15 \mathrm{~mm}$ in thickness. Two types of PMG are applied for the magnetic levitation force simulation. The cross section of the symmetrical PMG is $90 \mathrm{~mm}$ in width and

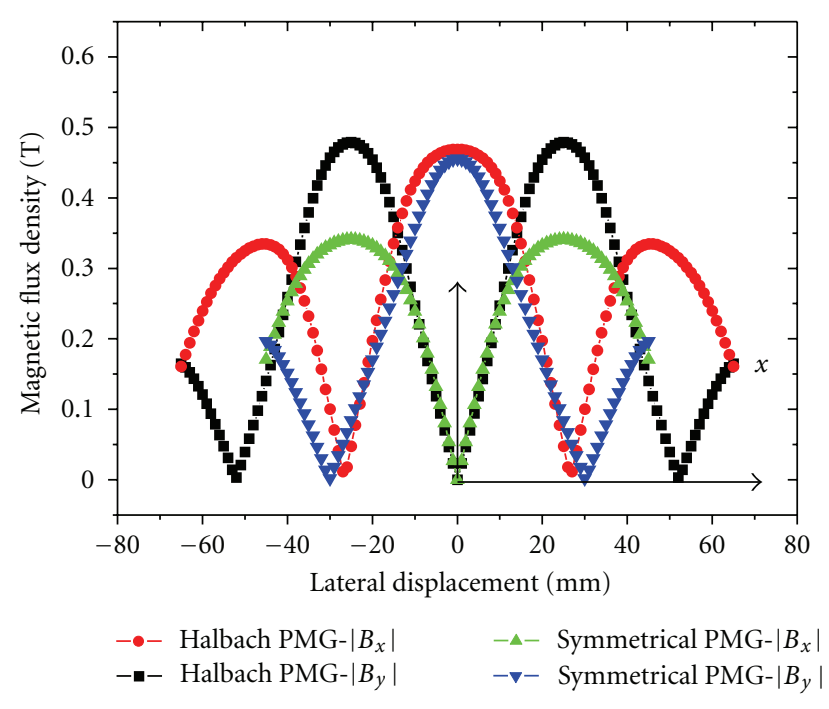

FIgure 3: The magnetic flux density distribution of Halbach PMG symmetrical PMG.

$40 \mathrm{~mm}$ in height. The cross section of the Halbach PMG is 80 in width and $20 \mathrm{~mm}$ in height. The standard analysis method to the equivalent surface current model is used to calculate the magnetic fields generated by PMG. The magnetization of $\mathrm{PM}$ is of $89500 \mathrm{~A} / \mathrm{m}^{2}$. Figure 3 shows the magnetic flux density distribution above the two types of PMG with the gap equal to $3 \mathrm{~mm}$.

The magnetic levitation force-gap loops are simulated with different lateral offsets. Figure 2 shows the schematic diagram of the simulation progress. During the simulation, the bulk HTS is in zero-field cooling condition. Firstly, the sample is vertically brought down from the position A to position $\mathrm{B}$ with specific lateral offset above the PMG. The moving velocity of the bulk HTS is equal to $1 \mathrm{~mm} / \mathrm{s}$. After the bulk sample arrives at position B, it is brought away back to position A with the same velocity. During the simulation progress, the lateral offset changes from $0 \mathrm{~mm}$ to $30 \mathrm{~mm}$ with the step size equal to $5 \mathrm{~mm}$. Each lateral offset step corresponds to one magnetic levitation force-gap loop.

The magnetic guidance forces are also calculated. Different from the levitation forces calculation, the bulk HTS was cooled in field-cooling condition. The sample was firstly moved to the position which is about $3 \mathrm{~mm}$ to the top surface of the PMG. It is called a field-cooling height $(\mathrm{CH})$. When the bulk HTS was cooled and changed into superconducting state, it started to move laterally. During the process, the gap between the bulk sample and the PMG equals to $3 \mathrm{~mm}$. It is called working height $(\mathrm{WH})$. The guidance force simulation was carried by moving the bulk HTS along $x$-axes.

Because of flux pinning potential ability, in experiment, the bulk HTS could freeze some magnetic flux inner. Mathematically, each mesh node's magnetic field of the sample was initialized with the applied magnetic fields. By the method, the guidance force was successfully calculated. 


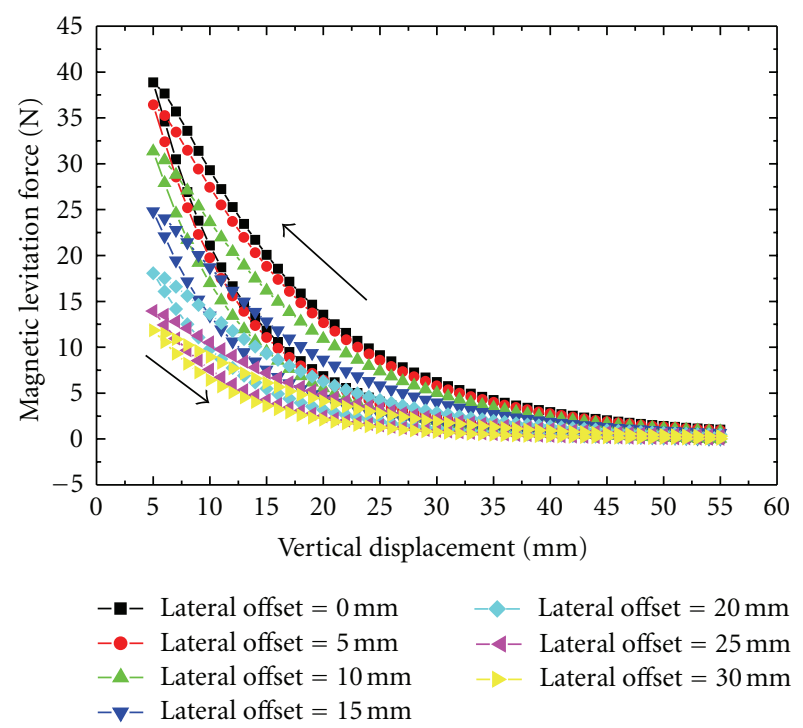

Figure 4: Magnetic levitation force loops of bulk HTS over symmetrical PMG with different lateral offsets.

\section{Simulation Results and Discussion}

Based on the proposed numerical method, the magnetic levitation force-gap loops of bulk HTS over PMG with different lateral offsets are obtained. The calculation parameters are of $M_{0}=89500 \mathrm{~A} / \mathrm{m}^{2}, J_{c}=8 \times 10^{7} \mathrm{~A} / \mathrm{m}^{2}$, and $E_{c}=1.0 \times$ $10^{-4} \mathrm{~V} / \mathrm{m}$. Figures 4 and 5 show the simulation results of bulk HTS over symmetrical PMG and Halbach PMG, respectively. From the results, we can see that all the levitation force-gap loops exhibit some hysteresis. This can be explained by the characteristics of HTS magnetic hysteretic effects. Figure 4 shows that with the decrease of the gap, the difference of levitation forces which corresponding to different lateral offset, of the bulk sample becomes larger. Furthermore, for the same gap, the levitation forces decrease with the increase of lateral offset of the bulk sample. The difference of levitation forces becomes larger while the lateral offset becomes larger. Figure 4 also shows that all force-gap loops are located in those regions which are above the levitation force zero line.

Figure 5 shows that all the levitation force-gap loops exhibit some hysteresis, moreover, with the increase of lateral offset, the hysteresis of force-gap loops becomes larger when the lateral offset changes from $0 \mathrm{~mm}$ to $25 \mathrm{~mm}$. When the lateral offset is larger than $25 \mathrm{~mm}$, the hysteresis of forcegap loop decreases instead. Figure 5 also shows that when the bulk HTS arrives at the low position B and begins moving vertically away back to position $\mathrm{A}$, the levitation forces become smaller and smaller. When the gap between the bottom of the bulk sample and the top surface of the PMG is larger than $17 \mathrm{~mm}$, the values of levitation force are negative which means the bulk HTS and PMG now exhibit attraction instead of repulsion.

Figure 6 shows the maximum value curves of the levitation force of bulk HTS over two types of PMG with

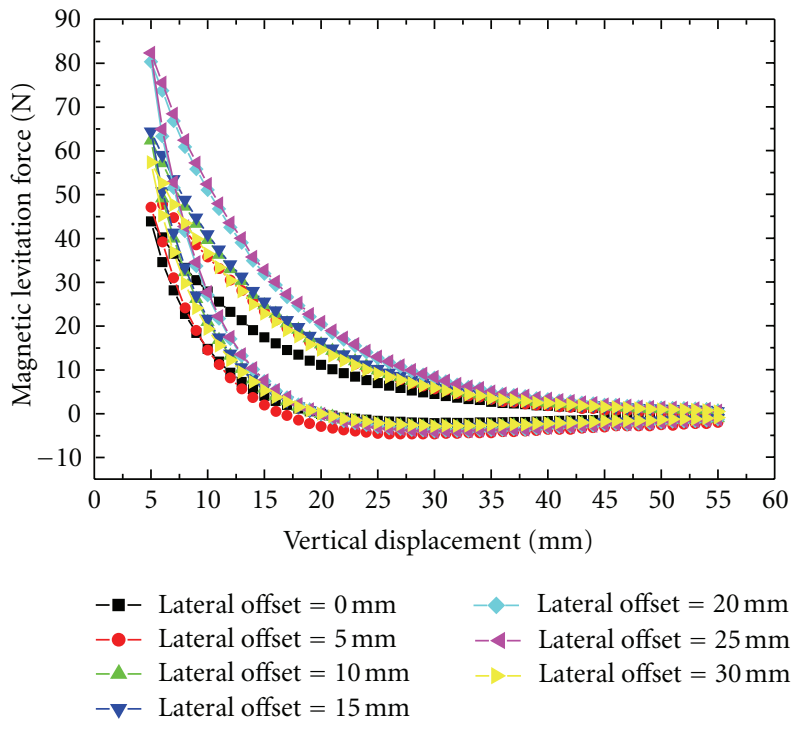

Figure 5: Magnetic levitation force loops of bulk HTS over Halbach PMG with different lateral offset.

different lateral offsets. From Figure 6 we can see that for symmetrical PMG, the maximum levitation forces of bulk HTS will decrease monotonously with the increasing of lateral offset. If the bulk HTS is above Halbach PMG, the maximum levitation forces of the bulk sample change nonmonotonously with the increasing of lateral offset. When the lateral offset changes from $0 \mathrm{~mm}$ to $25 \mathrm{~mm}$, the maximum levitation forces of the bulk HTS over Halbach PMG increase monotonously; when the lateral offset exceeding $25 \mathrm{~mm}$, the maximum levitation forces decrease rapidly. For the curve of maximum levitation force of bulk HTS over Halbach PMG, the maximum levitation force of bulk HTS with lateral offset equal to $25 \mathrm{~mm}$ is larger $88.6 \%$ than the minimum force of the bulk HTS which its lateral offset is equal to $0 \mathrm{~mm}$.

Figure 7 shows the guidance force-displacement curves of the bulk HTS over two types PMG. The negative guidance forces mean that it tries to pull the bulk HTS back to its initial position when the bulk sample is moved away the balance position. The calculation results show that the guidance force of the bulk HTS above the Halbach PMG is larger than that above the symmetrical PMG with the same lateral displacement. With the increase of the lateral displacement, the recover forces of the bulk HTS over the Halbach PMG increase faster than the bulk HTS over the symmetrical PMG.

The PMG is assumed infinite-long in $z$-axes. The magnetic fields only distribute in $x$ - $y$ plane. The magnetic fields $z$-axes component $B_{z}$ induced by the PMG is equal to zero. According to formula (10) the guidance force can be given by

$$
F_{\text {guid }}=B_{z} J_{y}-B_{y} J_{z}=-B_{y} J_{z}
$$

Here negative sign means the recover forces. From the magnetic fields distribution scheme Figure 3 we can see that when the lateral displacement increases and is greater than $5 \mathrm{~mm}$, the component $B_{y}$ induced by the symmetrical PMG 


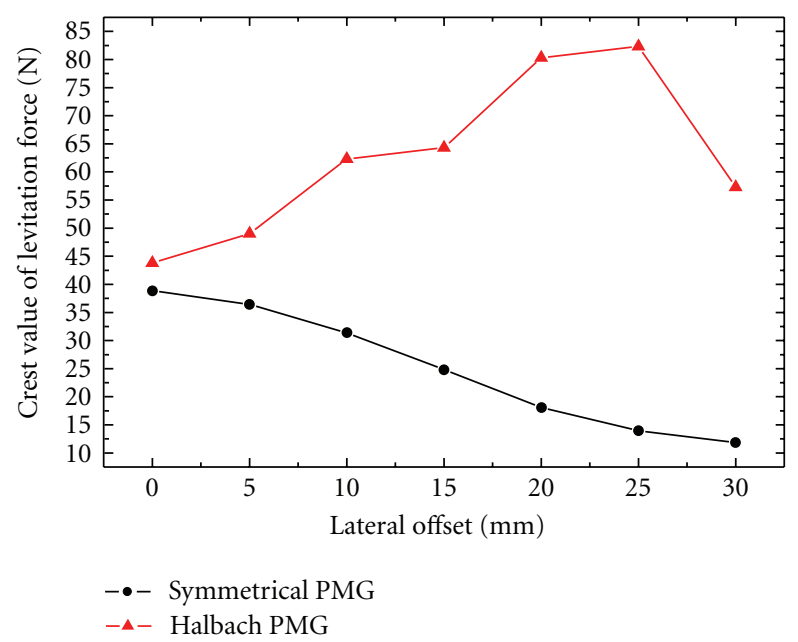

FIGURe 6: Maximum levitation forces of bulk HTS above two types PMG with different lateral offsets.

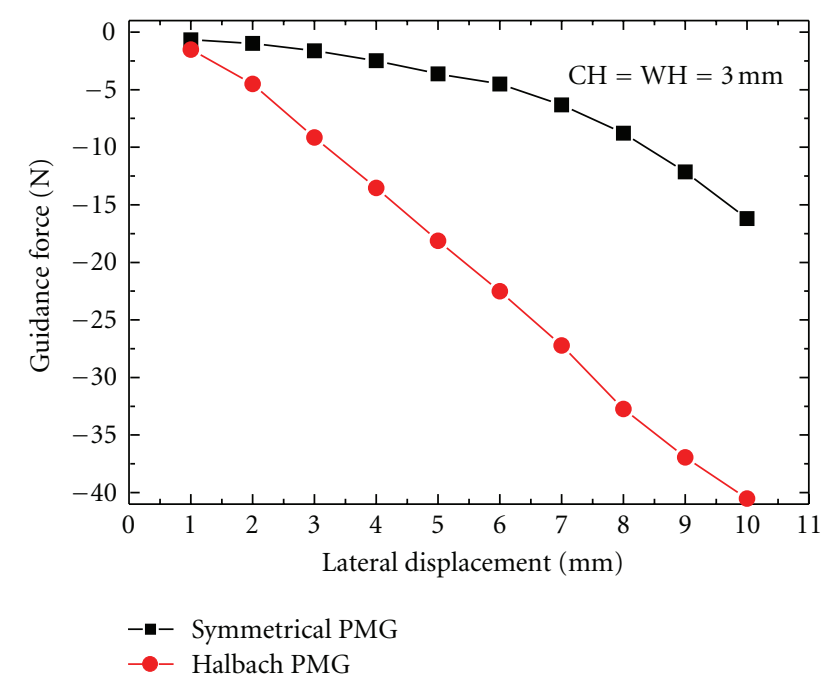

FIGURE 7: Guidance force-displacement curves of bulk HTS over two types PMG along $x$-axes.

reduces rapidly. On the contrary, the component $B_{y}$ induced by the Halbach PMG increases faster. This may be the main reason of the fact that the guidance force of the bulk HTS over the Halbach PMG is greater than the guidance force of the bulk HTS over the symmetrical PMG. Also there are some other factors such as the induced current density distribution inner the bulk HTS to influence the guidance forces performance.

From the discussion above we can get the conclusion that, in a Maglev system applied of symmetrical PMG, the bulk should be located close to the center of the PMG, which means HTS stagger array is better than side-by-side array arrangement (see Figures 1(a) and 1(b)). On the contrary, for Maglev system applied of Halbach PMG, the bulk HTS sideby-side array is better than stagger array and forth more, bulk HTS should be located close to the position of lateral offset equal to $25 \mathrm{~mm}$ instead of the center the Halbach PMG.

\section{Conclusion}

In the paper, levitation forces of cylindrical bulk HTS with different lateral offsets over two types of PMG are numerically presented. The simulation results show that the levitation force will decrease monotonously with the increase of lateral offset of the bulk HTS over symmetrical PMG.

For Halbach PMG, there is a value of the lateral offset of the bulk over the PMG, when the lateral offset changes from $0 \mathrm{~mm}$ to the value, the levitation force will increase monotonously; when the lateral offset exceeds the value, the levitation force decreases rapidly. From the simulation results we can get the conclusion that the optimization of magnetic levitation transportation system composed of bulk HTS and PMG not only consider the applied magnetic field enhancement, but also the applied magnetic field distribution. For symmetrical PMG, the bulk HTS stagger array arrangement may be better than others, for Halbach PMG, the bulk HTS side-by-side array is better than the stagger array arrangement.

\section{Acknowledgment}

This work is supported by the National Natural Science Foundation in China (no. 11205080).

\section{References}

[1] J. S. Wang, S. Y. Wang, Y. Zeng et al., "The first man-loading high temperature superconducting Maglev test vehicle in the world," Physica C, vol. 378-381, no. 1, pp. 809-814, 2002.

[2] M. Murakami, "Processing and applications of Bulk RE$\mathrm{Ba}-\mathrm{Cu}-\mathrm{O}$ superconductors," International Journal of Applied Ceramic Technology, vol. 4, no. 3, pp. 225-241, 2007.

[3] L. Schultz, O. de Haas, P. Verges et al., "Superconductively levitated transport system-the SupraTrans project," IEEE Transactions on Applied Superconductivity, vol. 15, no. 2, pp. 2301-2305, 2005.

[4] K. L. Kovalev, S. M. Koneev, V. N. Poltavec et al., "Magnetically levitated high-speed carriages on the basis of bulk HTS elements," in Proceedings of the 8th International Symposium on Magnetic Suspension Technology, pp. 45-49, Dresden, Germany, September 2005.

[5] W. J. Yang, M. Qiu, Y. Liu, Z. Wen, Y. Duan, and X. Chen, "Levitation characteristics in an HTS maglev launch assist test vehicle," Superconductor Science and Technology, vol. 20, no. 3, article 281, 2007.

[6] G. D'Ovidio, F. Crisi, and G. Lanzara, "A "V" shaped superconducting levitation module for lift and guidance of a magnetic transportation system," Physica $C$, vol. 468, no. 14, pp. 1036-1040, 2008.

[7] Z. Deng, J. Wang, J. Zheng et al., "High-efficiency and lowcost permanent magnet guideway consideration for high$T_{c}$ superconducting Maglev vehicle practical application," Superconductor Science and Technology, vol. 21, no. 11, Article ID 115018, 2008.

[8] X. Y. Zhang, Y. H. Zhou, and J. Zhou, "Influences of permanent magnets temperature characteristic on the levitation force of $\mathrm{YBaCuO}$ bulk superconductors," Journal of Superconductivity and Novel Magnetism, vol. 25, no. 4, pp. 857-860, 2012. 
[9] X. Y. Zhang, J. Zhou, and Y. H. Zhou, "Jump properties of the tip magnetic field of a notch in a melt-processes yttriumbarium-copper-oxide bulk," Journal of Applied Physics, vol. 107, no. 3, Article ID 036102, 3 pages, 2010.

[10] X. Y. Zhang, J. Zhou, and Y. H. Zhou, "Levitation properties of melt-processed YBCO bulk with a linear notch," Journal of Superconductivity and Novel Magnetism, vol. 23, no. 2, pp. 265-268, 2010.

[11] M. Liu, Y. Lu, S. Wang, and G. Ma, "Influence of AC external magnetic field on the levitation force relaxation of HTS bulk above NdFeB guideway," Journal of Superconductivity and Novel Magnetism, vol. 24, no. 5, pp. 1809-1813, 2011.

[12] M. Liu, Y. Lu, G. Ma, and S. Wang, "Oscillation characteristics of levitation force of YBCO bulk exposed to AC magnetic field above NdFeB guideway," Journal of Superconductivity and Novel Magnetism, vol. 24, no. 4, pp. 1339-1344, 2011.

[13] M. Liu, Y. Lu, S. Wang, and G. Ma, "Decay characteristics of levitation force of YBCO bulk exposed to AC magnetic field above NdFeB guideway," Journal of Low Temperature Physics, vol. 163, no. 1-2, pp. 78-85, 2011.

[14] Y. Lu, Y. Ge, M. Liu, and J. Wang, "High-performance permanent magnet railway design consideration of magnetic stiffness parameters of YBCO bulk arrays," Journal of Superconductivity and Novel Magnetism, vol. 24, no. 5, pp. 17871791, 2011.

[15] Y. Lu, J. Wang, S. Wang, and J. Zheng, "3D-modeling numerical solutions of electromagnetic behavior of HTSC bulk above permanent magnetic guideway," Journal of Superconductivity and Novel Magnetism, vol. 21, no. 8, pp. 467-472, 2008. 

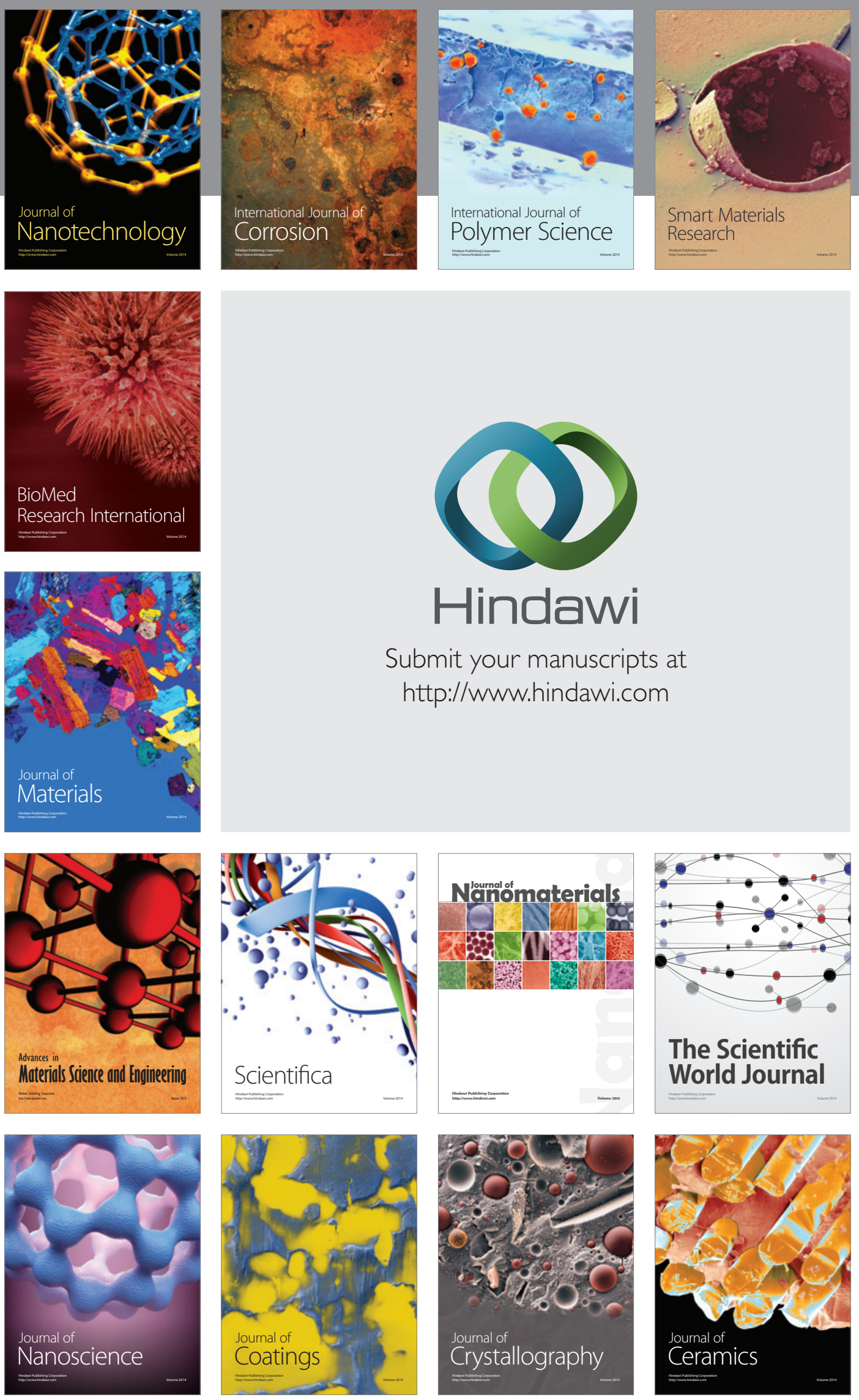

The Scientific World Journal

Submit your manuscripts at

http://www.hindawi.com

\section{World Journal}

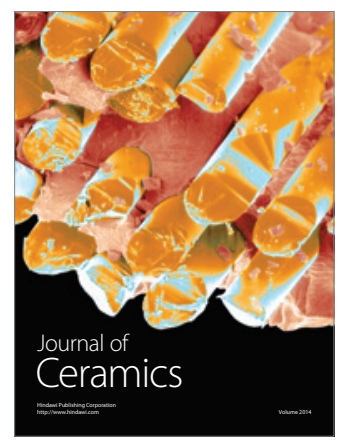

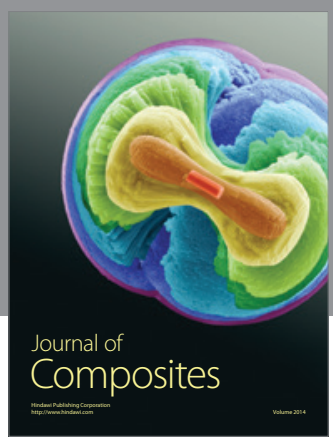
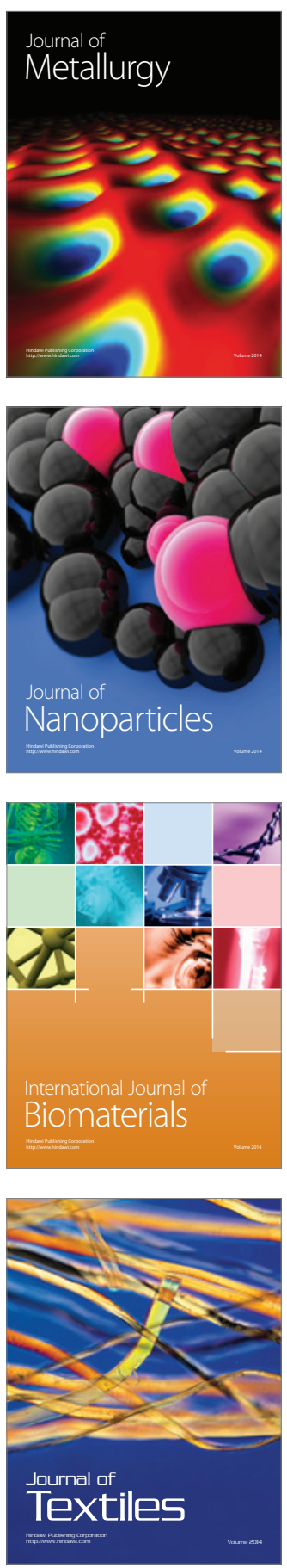